\title{
МЕТОД ОБНАРУЖЕНИЯ АРТЕФАКТОВ ПОТЕРИ ДАННЫХ В ИЗОБРАЖЕНИЯХ
}

\section{METHOD FOR DETECTING DATA LOSS ARTIFACTS IN IMAGES}

\section{A. Ustin}

Summary:The article presents a method for detecting data loss artifacts in images, which allows you to detect not only the artifact itself, or the probability of finding it in the images, but also the coordinates of this artifact. Algorithms such as NPBM allow obtaining only the image metric, in the form of a characteristic of the probability of finding artifacts in the image, which complicates both the diagnosis of problems associated with the algorithm and the assessment of its effectiveness. At the same time, obtaining the exact coordinates of artifacts allows you to search and correct these problems using third-party tools.

Keywords: image processing, quality assessment, video, data loss artifacts, algorithml.

\section{Введение}

$\mathrm{B}$ этой статье рассматривается проблема обнаружения артефактов потери данных в изображениях. Эта проблема часто встречается при потоковой передаче видео через различных средства связи и появляется при потерях в поступающем сигнале. В последнее десятилетие, в связи с обширным ростом пропускной способности сети интернет и ростом медиаконтента, растут и требования к такому контенту среди зрителей и как следствие, телерадиовещательных компаний. Вследствие этого, появляются запросы на создание систем и алгоритмов для проверки качества изображения, и по обнаружению в них дефектов. Одним из таких дефектов, являются рассматриваемые в этой статье артефакты потери данных. Данный вид дефектов проявляется в виде геометрического артефакта в изображениях, в виде квадратного макроблока, размером 8х8 пикселей, который заполнен различными, часто случайными, цветами. В последние десятилетия появилось много алгоритмом для обнаружения подобных артефактов, но большинство из них требуют референсное видео [1][2]. Однако для современных систем оценки качества видео более оптимальны без-референсные алгоритмы. На данный момент существуют алгоритмы, которые отталкиваются от попыток понять работу человеческого зрения. Например алгоритм GBIM[3] вычисляет блочную метрику в виде межпиксельной разницы на границах блоков, применяя функцию взвешивания, для применения эффекта HVS (Human Visual System). Алгоритм NPBM[4] использу-

\author{
Устин Антон Михайлович \\ Аспирант, ФГБОУ ВПО «Мордовский Государственный \\ Университет им. Н.П. Огарёва» \\ Landgraf132@yandex.ru
}

Аннотация: В статье представлен метод обнаружения артефактов потери данных в изображениях, который позволяет обнаружить не только сам артефакт, или вероятность его нахождения на изображениях, но и координаты данного артефакта. Алгоритмы, такие как NPBM, позволяют получать лишь метрику изображения, в виде характеристики вероятности нахождения артефактов на изображении, что усложняет как и диагностику проблем, связанных с алгоритмом, так и оценку его эффективности. При этом получение точных координат артефактов позволяет производить поиск и исправление данных проблем сторонними инструментами.

Ключевые слова: обработка изображений, оценка качества, видео, артефакты потери данных, алгоритм.

ет локально адаптивный подход, однако требует значительных вычислительных затрат.

\section{Описание алгоритма}

Первым шагом, как и во многих алгоритмах обработки изображений, идет преобразование исходного изображения из цветового пространства RGB в цветовое пространство YUV по следующим формулам:

$$
\begin{gathered}
Y=K_{R} \cdot R+\left(1-K_{R}-K_{B}\right) \cdot G+K_{B} \cdot B \\
U=B-Y \\
V=R-Y
\end{gathered}
$$

Где $K_{R}$ и $K_{B}$ - хроматические коэффициенты, предлагаемые ВТ.601[5] и равные $K_{R}=0.299$, а $K_{B}=0.114$.

Далее выполняется нормировка значений пикселей в канале яркостной компоненте изображения (Y). Данная операция выполняется в блоках размером 8х8 пикселей, в соответствии с геометрическим описанием рассматриваемого артефакта. Нормировка значений заключается в замене значений всех пикселей в блоке на одно значение, равное среднему арифметическому значений всех пикселей. На рисунке 1 виден результат данного нормирования.

Согласно формуле 2 и 3, делаем свертку нормированного изображения с матрицей Е и I (4) соответственно. Матрица Е даст метрику всего макроблока, за исключением интересующей нас внутренней части по формуле 


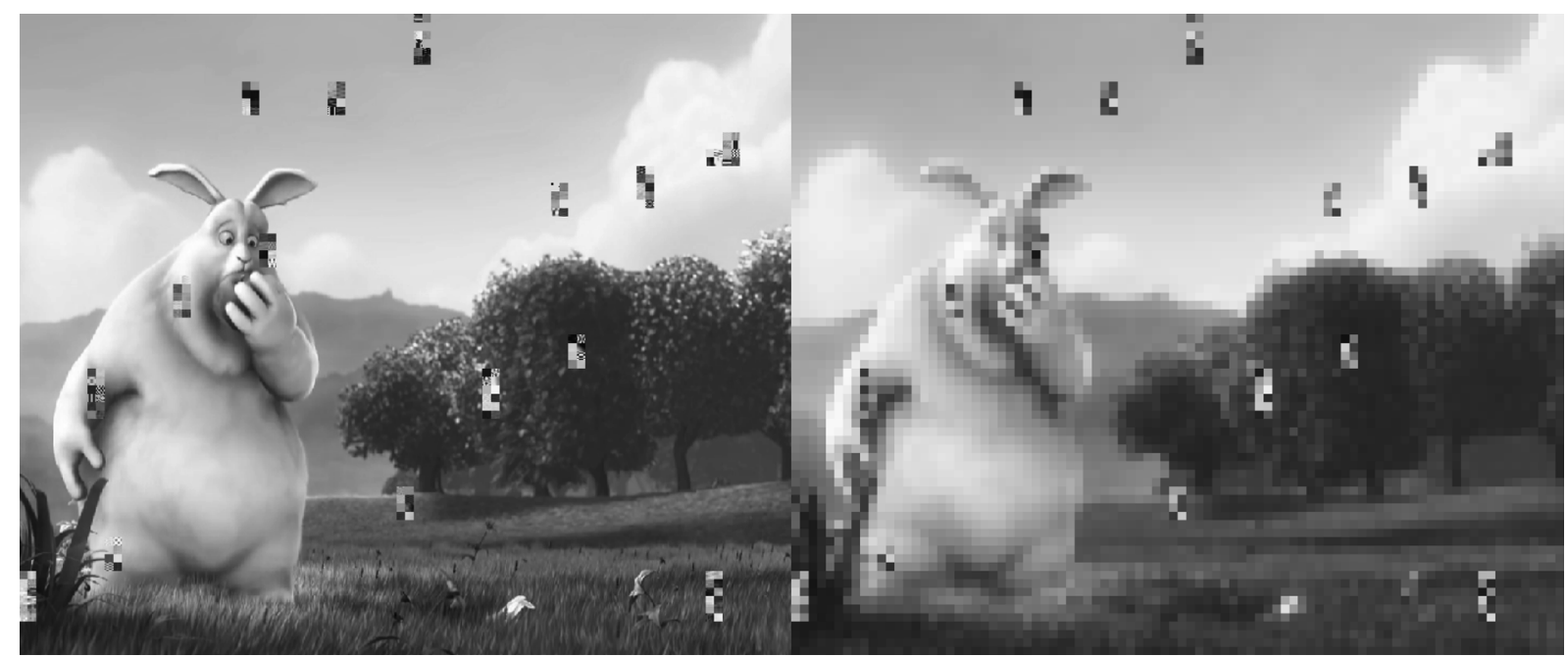

Рис. 1. Слева находится исходное изображение с артефактами потери данных, справа - яркостная компонента изображения с нормированными блоками 8х8 пикселей.

(5). Матрица I наоборот, даст метрику только интересующей нас части. Далее находим полную метрику всего макроблока, добавляя в неё среднее арифметическое внутреннего блока (6).

$$
\begin{aligned}
& \sum_{j=0}^{h} \sum_{i=0}^{w} x_{i, j} \cdot E_{m-i, n-j} \\
& \sum_{j=0}^{h} \sum_{i=0}^{w} x_{i, j} \cdot I_{m-i, n-j} \\
& E=\left[\begin{array}{llll}
1 & 1 & 1 & 1 \\
1 & 0 & 0 & 1 \\
1 & 0 & 0 & 1 \\
1 & 1 & 1 & 1
\end{array}\right] \\
& I=\left[\begin{array}{llll}
0 & 0 & 0 & 0 \\
0 & 1 & 1 & 0 \\
0 & 1 & 1 & 0 \\
0 & 0 & 0 & 0
\end{array}\right] \\
& R_{A}=\frac{\left(S_{I}+R_{I} \cdot 4\right)}{R_{E}=\frac{S_{E}}{16}} \\
& R_{I}=\frac{S_{I}}{16}
\end{aligned}
$$

Последним этапом находим отношение RA и RE и сравнивая с эмпирически подобранный порогом, получаем результирующую метрику для нахождения артефактов потери данных.

\section{Сравнение с Аругими алгоритмами}

Для оценки алгоритма, было выполнено сравнение произведенных метрик с результатами алгоритмов GBIM и NPBM. Тестирование было произведено на подготовленном наборе тестовых изображений, доступных по ссылке [6], результаты приведены на рисунке 2. Сравнение алгоритмов проводилось по следующим принципам:

Для созданного алгоритма корректно распознанным файлом считается то изображение, на котором верно распознан как минимум один артефакт потери данных, либо не распознано ничего, если само изображение их не содержит.

Для NPBM и GBIM были подобраны пороговые значения на исследуемом наборе данных и в зависимости от них делается вывод об обнаружении артефакта на изображении.

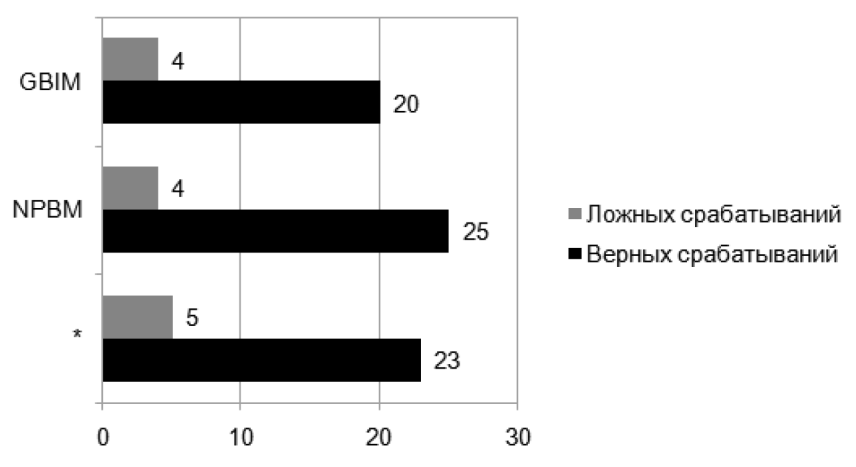

Рис. 2. Диаграмма результатов сравнения алгоритмов

\section{Скорость работы алгоритмов}

Тестирование проводилось на пяти файлах размером 


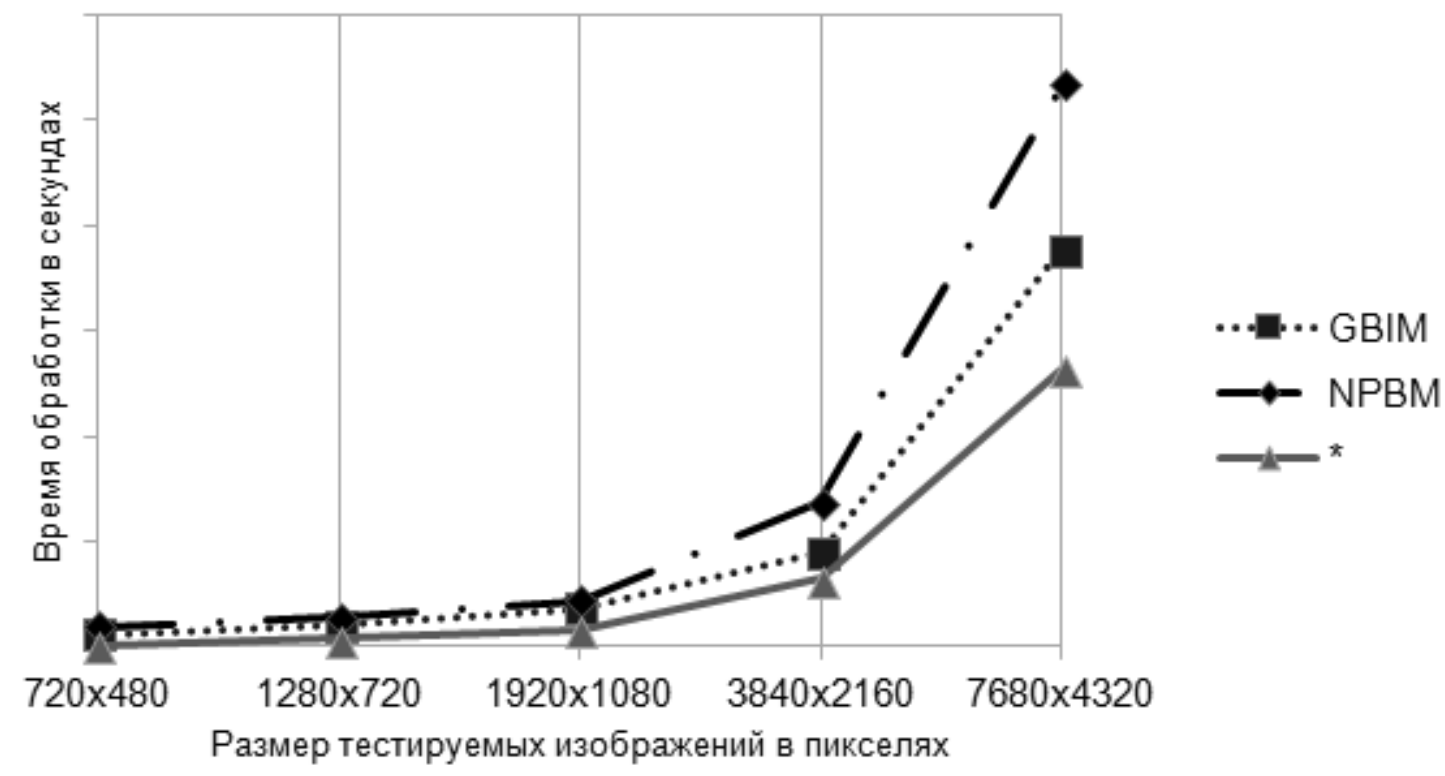

Рис. 3. Зависимость времени работы алгоритма от размера изображений

720x480, 1280x720, 1920x1080,3840×2160 и 7680x4320 точек. Характеристики оборудования: AMD Ryzen 7 2700X, 32 гБ DDR4 О3У, ОС: Ubuntu 20.04, компилятор: gсс 9.3.0.

На рисунке 3 видно, что разрабатываемый алгоритм показал наиболее быструю скорость обработки изобра- жений, что является важным преимуществом при использовании в потоковых системах проверки качества видео контента. При этом рассматриваемый алгоритм так же не сильно уступает алгоритму NPBM в качестве работы, что говорит о возможности успешного его внедрения и использования на практике.

\section{ЛИТЕРАТУРА}

1. Z.Yu, H.R. Wu, S. Winkler. Vision-ModelBased Impairment Metric to Evaluate Blocking Artifacts in Digital Video[Tekct] , /Proceedings of the IEEE, c. 154-169, 2002.

2. S.A. Karunasekera, N.G. Kingsbury. A Distortion Measure for Blocking Artifacts in Images Based on Human Visual Sensitivity[Tekct] /IEEE Trans. Image Processing, TOM. 4, H. 11, C. 713-724, 1995.

3. H.R. Wu and M. Yuen. A Generalized Block-edge Impairment Metric for Video Coding[Tekct] / IEEE Signal Processing Letters, ToM 70, н. 3, C. 247-278, 1998.

4. Hantao Liu, Ingrid Heynderickx. A NO-REFERENCE PERCEPTUAL BLOCKINESS METRIC[TekcT] / ICASSP 2008, c.865-868

5. Рекомендация МСЭ-R ВТ. 601-7.- Женева:ITU 2011. - 19 с.

6. Устин A.M. Ratevandr/dataLossArtifactGenerator [Электронный ресурс].- Режим доступа: https://github.com/Ratevandr/dataLossArtifactGenerator, свободный. Дата обращения [16.09.2020] 